OPEN $\curvearrowright$ ACCESS

드 COMMUNICATIONS

ISSN 2056-9890

\section{Crystal structure of bis(prop-2-yn-1-yl) 5-nitroisophthalate}

\author{
K. S. Ezhilarasi, ${ }^{\text {a }}$ Sivasamy Selvarani, ${ }^{\text {b }}$ Perumal \\ Rajakumar, ${ }^{\text {b }}$ B. K. Revathi ${ }^{\mathrm{a}}$ and G. Usha ${ }^{\mathrm{a} *}$
}

${ }^{\text {a } P G ~ a n d ~ R e s e a r c h ~ D e p a r t m e n t ~ o f ~ P h y s i c s, ~ Q u e e n ~ M a r y ' s ~ C o l l e g e, ~ C h e n n a i-4, ~}$ Tamilnadu, India, and ${ }^{\mathbf{b}}$ Department of Organic Chemistry, University of Madras, Guindy Campus, Chennai-25, India. *Correspondence e-mail: guqmc@yahoo.com

Received 12 May 2015; accepted 21 May 2015

Edited by H. Stoeckli-Evans, University of Neuchâtel, Switzerland

The whole molecule of the title compound, $\mathrm{C}_{14} \mathrm{H}_{9} \mathrm{NO}_{6}$, is generated by twofold rotation symmetry; the twofold axis bisects the nitro group and the benzene ring. The nitro group is inclined to the benzene ring by $14.42(9)^{\circ}$. The prop-2-yn-1yl groups are inclined to the benzene ring by $13(2)^{\circ}$ and to each other by $24(3)^{\circ}$; one directed above the plane of the benzene ring and the other below. In the crystal, molecules are linked via pairs of $\mathrm{C}-\mathrm{H} \cdots \mathrm{O}$ hydrogen bonds, forming inversion dimers with an $R_{2}^{2}(18)$ ring motif. The dimers are linked by further $\mathrm{C}-\mathrm{H} \cdots \mathrm{O}$ hydrogen bonds, forming sheets lying parallel to (100).

Keywords: crystal structure; 5-nitroisophthalate; prop-2-yn-1-yl; twofold rotation symmetry; $\mathrm{C}-\mathrm{H} \cdots \mathrm{O}$ hydrogen bonding.

CCDC reference: 1402145

\section{Related literature}

For the biological activities of carboxylates, see: Choudhary et al. (2002). For the uses and properties of nitroaromatics, see: Lee et al. (2013); Somerville et al. (1995).<smiles>C#CCOC(=O)c1cc(C(=O)OCC#C)cc([N+](=O)[O-])c1</smiles>

\section{Experimental}

2.1. Crystal data

$\mathrm{C}_{14} \mathrm{H}_{9} \mathrm{NO}_{6}$

$M_{r}=287.22$

Orthorhombic, Pccn

$a=6.679(5) \AA$

$b=11.679(5) \AA$

$c=16.503(5) \AA$

$V=1287.3(12) \AA^{3}$

$Z=4$

Mo $K \alpha$ radiation

$\mu=0.12 \mathrm{~mm}^{-1}$

$T=293 \mathrm{~K}$

$0.30 \times 0.25 \times 0.20 \mathrm{~mm}$

\subsection{Data collection}

Bruker Kappa APEXII CCD diffractometer

Absorption correction: multi-scan

(SADABS; Bruker, 2008)

$T_{\min }=0.965, T_{\max }=0.977$

6369 measured reflections 1613 independent reflections 1316 reflections with $I>2 \sigma(I)$ $R_{\text {int }}=0.021$

\subsection{Refinement}

$R\left[F^{2}>2 \sigma\left(F^{2}\right)\right]=0.038$

$w R\left(F^{2}\right)=0.136$

$S=0.73$

1523 reflections

98 parameters

$\mathrm{H}$-atom parameters constrained

$\Delta \rho_{\max }=0.26 \mathrm{e} \AA^{-3}$

$\Delta \rho_{\min }=-0.21 \mathrm{e} \AA^{-3}$

Table 1

Hydrogen-bond geometry $\left(\AA,{ }^{\circ}\right)$.

\begin{tabular}{lllll}
\hline$D-\mathrm{H} \cdots A$ & $D-\mathrm{H}$ & $\mathrm{H} \cdots A$ & $D \cdots A$ & $D-\mathrm{H} \cdots A$ \\
\hline $\mathrm{C} 6-\mathrm{H} 6 A \cdots \mathrm{O} 1^{\mathrm{i}}$ & 0.97 & 2.46 & $3.334(2)$ & 150 \\
$\mathrm{C} 6-\mathrm{H} 6 B \cdots 1^{\mathrm{ii}}$ & 0.97 & 2.57 & $3.313(2)$ & 134 \\
$\mathrm{C} 8-\mathrm{H} 8 \cdots \mathrm{O}^{\mathrm{ii}}$ & 0.93 & 2.50 & $3.251(2)$ & 138 \\
\hline
\end{tabular}

Symmetry codes: (i) $-x+2,-y+1,-z$; (ii) $-x+\frac{5}{2}, y, z-\frac{1}{2}$.

Data collection: APEX2 (Bruker, 2008); cell refinement: SAINT (Bruker, 2008); data reduction: SAINT; program(s) used to solve structure: SHELXS97 (Sheldrick, 2008); program(s) used to refine structure: SHELXL97 (Sheldrick, 2008); molecular graphics: ORTEP-3 for Windows (Farrugia, 2012); software used to prepare material for publication: SHELXL97 and PLATON (Spek, 2009).

\title{
Acknowledgements
}

The authors thank the SAIF, IIT Madras, for providing the $\mathrm{X}$-ray data-collection facility.

Supporting information for this paper is available from the IUCr electronic archives (Reference: SU5137).

\section{References}

Bruker (2008). APEX2, SAINT and SADABS. Bruker AXS Inc., Madison, Wisconsin, USA.

Choudhary, M. A., Mazhar, M., Ali, S., Song, X. \& Eng, G. (2002). Met.-Based Drugs, 8, 275-281.

Farrugia, L. J. (2012). J. Appl. Cryst. 45, 849-854.

Lee, C. H., Kim, S. H., Kwon, D. H., Jang, K. H., Chung, Y. H. \& Moon, J. D. (2013). Ann. Occup. Environ. Med. 25, 31.

Sheldrick, G. M. (2008). Acta Cryst. A64, 112-122.

Somerville, C. C., Nishino, F. S. \& Spain, C. J. (1995). J. Bacteriol. 177, $3837-$ 3842.

Spek, A. L. (2009). Acta Cryst. D65, 148-155. 


\section{supporting information}

Acta Cryst. (2015). E71, o435 [doi:10.1107/S2056989015009846]

\section{Crystal structure of bis(prop-2-yn-1-yl) 5-nitroisophthalate}

\section{K. S. Ezhilarasi, Sivasamy Selvarani, Perumal Rajakumar, B. K. Revathi and G. Usha}

\section{S1. Comments}

Carboxylates have promising activity against various antitumor cells (Choudharyl et al., 2002). Nitroaromatic compounds are used in the production of dyes, plastics, high explosives, pharmaceuticals, and pesticides (Somerville et al., 1995). Nitrobenzene is mostly used in the synthesis of aniline and in the production of benzidine, quinolone and azobenzene (Lee et al., 2013).

In the title compound, Fig. 1, the two-fold rotation bisects the benzene ring and the nitro group; atoms $\mathrm{C} 1, \mathrm{C} 4, \mathrm{H} 4$ and $\mathrm{N} 1$ lie on the two-fold rotation axis. The nitro group is inclined to the benzene ring by 14.42 (9) ${ }^{\circ}$. The prop-2-yn-1-yl groups are inclined to the benzene ring by 13 (2) ${ }^{\circ}$ and to each other by 24 (3) ${ }^{\circ}$; one directed above the plane of the benzene ring and the other below.

In the crystal, molecules are linked via pairs of $\mathrm{C}-\mathrm{H} \cdots \mathrm{O}$ hydrogen bonds forming inversion dimers with an $R_{2}{ }^{2}(18)$ ring motif (Table 1). The dimers are linked by further $\mathrm{C}-\mathrm{H} \cdots \mathrm{O}$ hydrogen bonds forming sheets lying parallel to (100); see Table 1 and Fig. 2.

\section{S2. Synthesis and crystallization}

The title compound was synthesized by Steglich esterification of 5-nitro isophthalic acid (1 equiv) which together with propargyl alcohol (2.2 equiv) was added at $273 \mathrm{~K}$ to DMAP (2.5 equiv) and DCC ( 2.2 equiv) in dichloromethane (100 $\mathrm{ml}$ ). The mixture was stirred under nitrogen at room temperature for $24 \mathrm{~h}$. The white precipitate that formed was filtered off and washed with DCM $(150 \mathrm{ml})$ and brine $(150 \mathrm{ml})$, then dried over $\mathrm{Na}_{2} \mathrm{SO}_{4}$, filtered and evaporated to afforded the title compound. It was purified by column chromatography using $\mathrm{CHCl}_{3}$ :hexane $(9: 1)$ as a eluent. Crystals were obtained by slow evaporation of the solvent.

\section{S3. Refinement}

Crystal data, data collection and structure refinement details are summarized in Table 2. $\mathrm{H}$ atoms were positioned geometrically and treated as riding atoms: $\mathrm{C}-\mathrm{H}=0.93-0.97 \AA$ with $\mathrm{U}_{\mathrm{iso}}(\mathrm{H})=1.5 \mathrm{U}_{\mathrm{eq}}(\mathrm{C})$ for methyl $\mathrm{H}$ atoms and $1.2 \mathrm{U}_{\mathrm{eq}}(\mathrm{C})$ for other $\mathrm{H}$ atoms. 


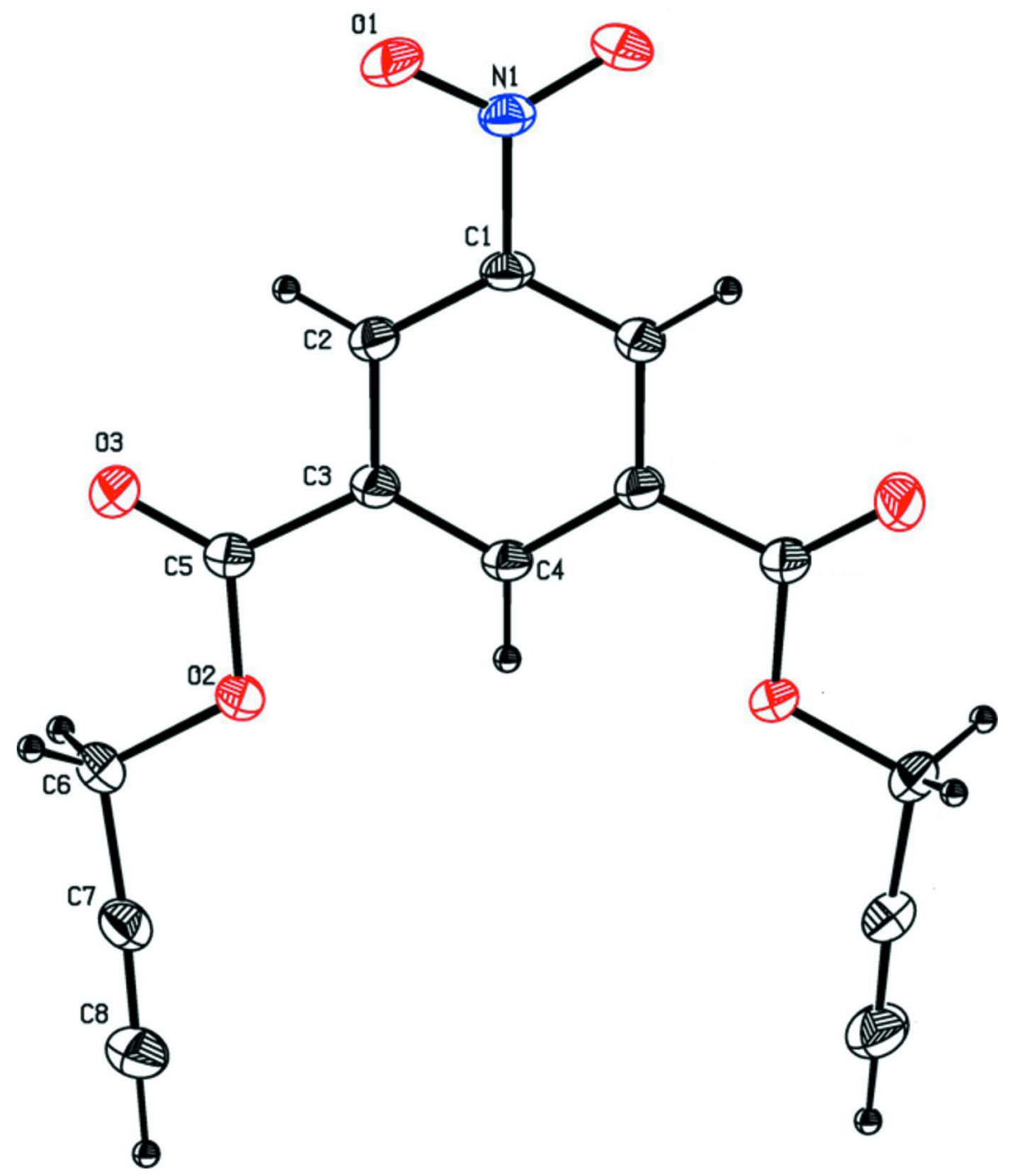

\section{Figure 1}

The molecular structure of the title compound, with atom labelling. Displacement ellipsoids are drawn at the 30\% probability level. The unlabelled atoms are related to the labelled atoms by twofold rotation symmetry [symmetry code: (i) $-x+3 / 2,-y+1 / 2, z]$. 


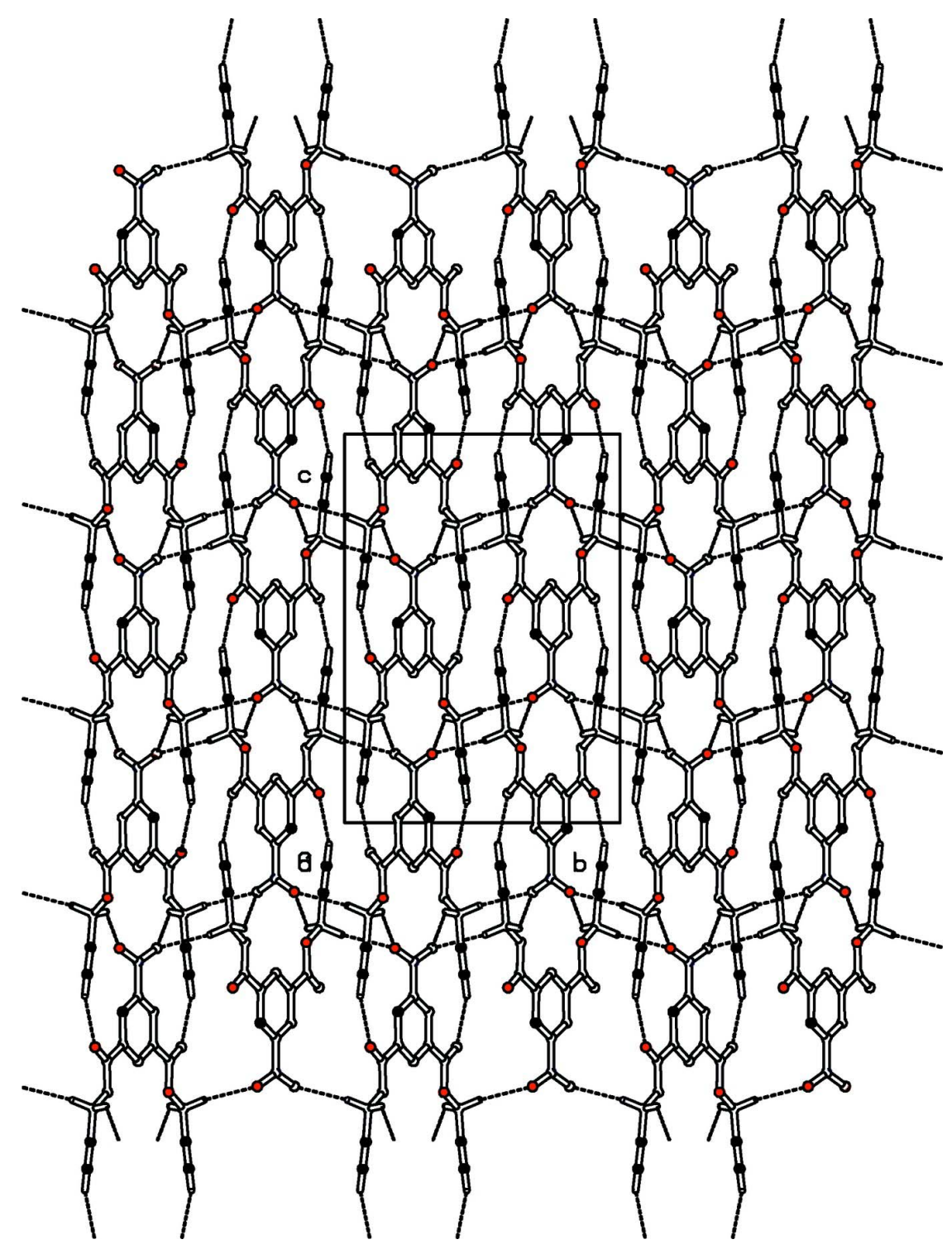

Figure 2

A view along the $a$ axis of the crystal packing of the title compound. The dashed lines indicate hydrogen bonds (see Table 1 for details).

Bis(prop-2-yn-1-yl) 5-nitrobenzene-1,3-dicarboxylate

Crystal data

$\mathrm{C}_{14} \mathrm{H}_{9} \mathrm{NO}_{6}$

$M_{r}=287.22$

Orthorhombic, Pccn

Hall symbol: -P 2ab 2ac

$a=6.679(5) \AA$

$b=11.679(5) \AA$

$c=16.503(5) \AA$

$V=1287.3(12) \AA^{3}$

$Z=4$
$F(000)=592$

$D_{\mathrm{x}}=1.482 \mathrm{Mg} \mathrm{m}^{-3}$

Mo $K \alpha$ radiation, $\lambda=0.71073 \AA$

$\theta=2.5-28.4^{\circ}$

$\mu=0.12 \mathrm{~mm}^{-1}$

$T=293 \mathrm{~K}$

Block, colourless

$0.30 \times 0.25 \times 0.20 \mathrm{~mm}$ 


\section{Data collection}

Bruker Kappa APEXII CCD diffractometer

Radiation source: fine-focus sealed tube Graphite monochromator

$\omega$ and $\varphi$ scan

Absorption correction: multi-scan

(SADABS; Bruker, 2008)

$T_{\min }=0.965, T_{\max }=0.977$

\section{Refinement}

Refinement on $F^{2}$

Least-squares matrix: full

$R\left[F^{2}>2 \sigma\left(F^{2}\right)\right]=0.038$

$w R\left(F^{2}\right)=0.136$

$S=0.73$

1523 reflections

98 parameters

0 restraints

Primary atom site location: structure-invariant direct methods

Secondary atom site location: difference Fourier map

\section{Special details}

Geometry. All e.s.d.'s (except the e.s.d. in the dihedral angle between two 1.s. planes) are estimated using the full covariance matrix. The cell e.s.d.'s are taken into account individually in the estimation of e.s.d.'s in distances, angles and torsion angles; correlations between e.s.d.'s in cell parameters are only used when they are defined by crystal symmetry. An approximate (isotropic) treatment of cell e.s.d.'s is used for estimating e.s.d.'s involving 1.s. planes.

Refinement. Refinement of $F^{2}$ against ALL reflections. The weighted $R$-factor $w R$ and goodness of fit $S$ are based on $F^{2}$, conventional $R$-factors $R$ are based on $F$, with $F$ set to zero for negative $F^{2}$. The threshold expression of $F^{2}>\sigma\left(F^{2}\right)$ is used only for calculating $R$-factors(gt) $e t c$. and is not relevant to the choice of reflections for refinement. $R$-factors based on $F^{2}$ are statistically about twice as large as those based on $F$, and $R$ - factors based on ALL data will be even larger.

Fractional atomic coordinates and isotropic or equivalent isotropic displacement parameters $\left(\hat{A}^{2}\right)$

\begin{tabular}{lllll}
\hline & $x$ & $y$ & $z$ & $U_{\text {iso }} / U_{\text {eq }}$ \\
\hline C1 & 0.7500 & 0.2500 & $0.05352(9)$ & $0.0336(4)$ \\
C2 & $0.90289(17)$ & $0.30592(10)$ & $0.01386(7)$ & $0.0351(3)$ \\
H2 & 1.0033 & 0.3433 & 0.0426 & $0.042^{*}$ \\
C3 & $0.90264(16)$ & $0.30486(10)$ & $-0.07063(7)$ & $0.0329(3)$ \\
C4 & 0.7500 & 0.2500 & $-0.11315(9)$ & $0.0328(4)$ \\
H4 & 0.7500 & 0.2500 & -0.1695 & $0.039^{*}$ \\
C5 & $1.07371(18)$ & $0.36348(11)$ & $-0.11169(7)$ & $0.0377(3)$ \\
C6 & $1.2259(2)$ & $0.41344(12)$ & $-0.23491(7)$ & $0.0431(3)$ \\
H6A & 1.2527 & 0.4888 & -0.2127 & $0.052^{*}$ \\
H6B & 1.3450 & 0.3669 & -0.2284 & $0.052^{*}$ \\
C7 & $1.17465(19)$ & $0.42270(11)$ & $-0.32014(8)$ & $0.0412(3)$ \\
C8 & $1.1422(2)$ & $0.43439(16)$ & $-0.38961(9)$ & $0.0565(4)$ \\
H8 & 1.1166 & 0.4436 & -0.4446 & $0.068^{*}$ \\
N1 & 0.7500 & 0.2500 & $0.14284(8)$ & $0.0377(4)$ \\
O1 & $0.86053(16)$ & $0.31731(9)$ & $0.17767(6)$ & $0.0525(3)$
\end{tabular}

6369 measured reflections

1613 independent reflections

1316 reflections with $I>2 \sigma(I)$

$R_{\text {int }}=0.021$

$\theta_{\max }=28.4^{\circ}, \theta_{\min }=2.5^{\circ}$

$h=-8 \rightarrow 8$

Hydrogen site location: inferred from

neighbouring sites

$\mathrm{H}$-atom parameters constrained

$w=1 /\left[\sigma^{2}\left(F_{\mathrm{o}}^{2}\right)+(0.1246 P)^{2}+0.5331 P\right]$

where $P=\left(F_{\mathrm{o}}^{2}+2 F_{\mathrm{c}}^{2}\right) / 3$

$(\Delta / \sigma)_{\max }<0.001$

$\Delta \rho_{\min }=-0.21$ e $\AA^{-3}$

Extinction correction: SHELXL97 (Sheldrick,

2008), $\mathrm{Fc}^{*}=\mathrm{kFc}\left[1+0.001 \mathrm{xFc}^{2} \lambda^{3} / \sin (2 \theta)\right]^{-1 / 4}$

Extinction coefficient: 0.030 (5) 
supporting information

\begin{tabular}{lllll}
$\mathrm{O} 3$ & $1.21118(18)$ & $0.40639(12)$ & $-0.07644(6)$ & $0.0700(4)$ \\
$\mathrm{O} 2$ & $1.05835(13)$ & $0.36103(8)$ & $-0.19240(5)$ & $0.0404(3)$ \\
\hline
\end{tabular}

Atomic displacement parameters $\left(\AA^{2}\right)$

\begin{tabular}{lllllll}
\hline & $U^{11}$ & $U^{22}$ & $U^{33}$ & $U^{12}$ & $U^{13}$ & $U^{23}$ \\
\hline C1 & $0.0357(8)$ & $0.0445(8)$ & $0.0205(8)$ & $0.0030(6)$ & 0.000 & 0.000 \\
C2 & $0.0344(6)$ & $0.0445(6)$ & $0.0263(6)$ & $-0.0024(4)$ & $-0.0023(4)$ & $-0.0013(4)$ \\
C3 & $0.0328(6)$ & $0.0407(6)$ & $0.0254(6)$ & $-0.0011(4)$ & $0.0013(4)$ & $0.0007(4)$ \\
C4 & $0.0355(8)$ & $0.0411(8)$ & $0.0218(7)$ & $-0.0010(6)$ & 0.000 & 0.000 \\
C5 & $0.0378(6)$ & $0.0483(7)$ & $0.0270(6)$ & $-0.0062(5)$ & $0.0000(5)$ & $0.0015(5)$ \\
C6 & $0.0392(7)$ & $0.0559(7)$ & $0.0342(7)$ & $-0.0110(5)$ & $0.0065(5)$ & $0.0047(5)$ \\
C7 & $0.0401(6)$ & $0.0463(6)$ & $0.0370(7)$ & $-0.0021(5)$ & $0.0090(5)$ & $0.0045(5)$ \\
C8 & $0.0549(8)$ & $0.0771(10)$ & $0.0376(7)$ & $-0.0064(7)$ & $0.0045(6)$ & $0.0094(7)$ \\
N1 & $0.0369(7)$ & $0.0529(8)$ & $0.0234(7)$ & $0.0042(6)$ & 0.000 & 0.000 \\
O1 & $0.0568(6)$ & $0.0728(7)$ & $0.0278(5)$ & $-0.0089(5)$ & $-0.0065(4)$ & $-0.0078(4)$ \\
O3 & $0.0606(7)$ & $0.1147(11)$ & $0.0345(5)$ & $-0.0461(7)$ & $-0.0069(5)$ & $0.0068(6)$ \\
O2 & $0.0393(5)$ & $0.0563(6)$ & $0.0256(5)$ & $-0.0124(4)$ & $0.0048(3)$ & $-0.0012(3)$ \\
& & & & & & \\
\hline
\end{tabular}

Geometric parameters $\left(A,{ }^{\circ}\right)$

\begin{tabular}{|c|c|c|c|}
\hline $\mathrm{C} 1-\mathrm{C} 2^{\mathrm{i}}$ & $1.3775(15)$ & $\mathrm{C} 5-\mathrm{O} 2$ & $1.3363(15)$ \\
\hline $\mathrm{C} 1-\mathrm{C} 2$ & $1.3775(15)$ & $\mathrm{C} 6-\mathrm{C} 7$ & $1.4517(18)$ \\
\hline $\mathrm{C} 1-\mathrm{N} 1$ & $1.474(2)$ & $\mathrm{C} 6-\mathrm{O} 2$ & $1.4555(15)$ \\
\hline $\mathrm{C} 2-\mathrm{C} 3$ & $1.3944(16)$ & C6-H6A & 0.9700 \\
\hline $\mathrm{C} 2-\mathrm{H} 2$ & 0.9300 & C6-H6B & 0.9700 \\
\hline $\mathrm{C} 3-\mathrm{C} 4$ & $1.3937(15)$ & $\mathrm{C} 7-\mathrm{C} 8$ & $1.175(2)$ \\
\hline $\mathrm{C} 3-\mathrm{C} 5$ & $1.4944(17)$ & $\mathrm{C} 8-\mathrm{H} 8$ & 0.9300 \\
\hline $\mathrm{C} 4-\mathrm{C} 3^{\mathrm{i}}$ & $1.3937(15)$ & $\mathrm{N} 1-\mathrm{O} 1$ & $1.2220(12)$ \\
\hline $\mathrm{C} 4-\mathrm{H} 4$ & 0.9300 & $\mathrm{~N} 1-\mathrm{O} 1^{\mathrm{i}}$ & $1.2220(12)$ \\
\hline $\mathrm{C} 5-\mathrm{O} 3$ & $1.1970(17)$ & & \\
\hline $\mathrm{C} 2 \mathrm{i}-\mathrm{C} 1-\mathrm{C} 2$ & $123.27(14)$ & $\mathrm{O} 2-\mathrm{C} 5-\mathrm{C} 3$ & $112.55(10)$ \\
\hline $\mathrm{C} 2 \mathrm{i}-\mathrm{C} 1-\mathrm{N} 1$ & $118.36(7)$ & $\mathrm{C} 7-\mathrm{C} 6-\mathrm{O} 2$ & $108.50(11)$ \\
\hline $\mathrm{C} 2-\mathrm{C} 1-\mathrm{N} 1$ & $118.36(7)$ & $\mathrm{C} 7-\mathrm{C} 6-\mathrm{H} 6 \mathrm{~A}$ & 110.0 \\
\hline $\mathrm{C} 1-\mathrm{C} 2-\mathrm{C} 3$ & $118.03(11)$ & $\mathrm{O} 2-\mathrm{C} 6-\mathrm{H} 6 \mathrm{~A}$ & 110.0 \\
\hline $\mathrm{C} 1-\mathrm{C} 2-\mathrm{H} 2$ & 121.0 & $\mathrm{C} 7-\mathrm{C} 6-\mathrm{H} 6 \mathrm{~B}$ & 110.0 \\
\hline $\mathrm{C} 3-\mathrm{C} 2-\mathrm{H} 2$ & 121.0 & $\mathrm{O} 2-\mathrm{C} 6-\mathrm{H} 6 \mathrm{~B}$ & 110.0 \\
\hline $\mathrm{C} 4-\mathrm{C} 3-\mathrm{C} 2$ & $120.56(11)$ & $\mathrm{H} 6 \mathrm{~A}-\mathrm{C} 6-\mathrm{H} 6 \mathrm{~B}$ & 108.4 \\
\hline $\mathrm{C} 4-\mathrm{C} 3-\mathrm{C} 5$ & $122.79(11)$ & $\mathrm{C} 8-\mathrm{C} 7-\mathrm{C} 6$ & $176.19(14)$ \\
\hline $\mathrm{C} 2-\mathrm{C} 3-\mathrm{C} 5$ & $116.64(10)$ & $\mathrm{C} 7-\mathrm{C} 8-\mathrm{H} 8$ & 180.0 \\
\hline $\mathrm{C} 3-\mathrm{C} 4-\mathrm{C} 3^{\mathrm{i}}$ & $119.53(14)$ & $\mathrm{O} 1-\mathrm{N} 1-\mathrm{O} 1^{\mathrm{i}}$ & $123.89(15)$ \\
\hline $\mathrm{C} 3-\mathrm{C} 4-\mathrm{H} 4$ & 120.2 & $\mathrm{O} 1-\mathrm{N} 1-\mathrm{C} 1$ & $118.06(7)$ \\
\hline $\mathrm{C} 3^{\mathrm{i}}-\mathrm{C} 4-\mathrm{H} 4$ & 120.2 & $\mathrm{O} 1{ }^{\mathrm{i}}-\mathrm{N} 1-\mathrm{C} 1$ & $118.06(7)$ \\
\hline $\mathrm{O} 3-\mathrm{C} 5-\mathrm{O} 2$ & $123.52(11)$ & $\mathrm{C} 5-\mathrm{O} 2-\mathrm{C} 6$ & $114.36(9)$ \\
\hline $\mathrm{O} 3-\mathrm{C} 5-\mathrm{C} 3$ & $123.92(12)$ & & \\
\hline $\mathrm{C} 2-\mathrm{C} 1-\mathrm{C} 2-\mathrm{C} 3$ & $-0.45(8)$ & $\mathrm{C} 2-\mathrm{C} 3-\mathrm{C} 5-\mathrm{O} 2$ & $-178.24(10)$ \\
\hline
\end{tabular}


supporting information

$\begin{array}{llll}\mathrm{N} 1-\mathrm{C} 1-\mathrm{C} 2-\mathrm{C} 3 & 179.55(8) & \mathrm{O} 2-\mathrm{C} 6-\mathrm{C} 7-\mathrm{C} 8 & 166(2) \\ \mathrm{C} 1-\mathrm{C} 2-\mathrm{C} 3-\mathrm{C} 4 & 0.91(15) & \mathrm{C} 2-\mathrm{C} 1-\mathrm{N} 1-\mathrm{O} 1 & -165.80(8) \\ \mathrm{C} 1-\mathrm{C} 2-\mathrm{C} 3-\mathrm{C} 5 & -178.32(9) & \mathrm{C} 2-\mathrm{C} 1-\mathrm{N} 1-\mathrm{O} 1 & 14.20(8) \\ \mathrm{C} 2-\mathrm{C} 3-\mathrm{C} 4-\mathrm{C} 3 & -0.46(8) & \mathrm{C} 2-\mathrm{C} 1-\mathrm{N} 1-\mathrm{O} 1^{\mathrm{i}} & 14.20(8) \\ \mathrm{C} 5-\mathrm{C} 3-\mathrm{C} 4-\mathrm{C} 3^{\mathrm{i}} & 178.71(12) & \mathrm{C} 2-\mathrm{C} 1-\mathrm{N} 1-\mathrm{O} 1^{\mathrm{i}} & -165.80(8) \\ \mathrm{C} 4-\mathrm{C} 3-\mathrm{C} 5-\mathrm{O} 3 & -176.32(13) & \mathrm{O} 3-\mathrm{C} 5-\mathrm{O} 2-\mathrm{C} 6 & 1.09(19) \\ \mathrm{C} 2-\mathrm{C} 3-\mathrm{C} 5-\mathrm{O} 3 & 2.9(2) & \mathrm{C} 3-\mathrm{C} 5-\mathrm{O} 2-\mathrm{C} 6 & -177.80(10) \\ \mathrm{C} 4-\mathrm{C} 3-\mathrm{C} 5-\mathrm{O} 2 & 2.56(15) & \mathrm{C} 7-\mathrm{C} 6-\mathrm{O} 2-\mathrm{C} 5 & -170.29(11)\end{array}$

Symmetry code: (i) $-x+3 / 2,-y+1 / 2, z$.

Hydrogen-bond geometry $\left(\AA,{ }^{\circ}\right)$

\begin{tabular}{lllll}
\hline$D-\mathrm{H} \cdots A$ & $D-\mathrm{H}$ & $\mathrm{H} \cdots A$ & $D \cdots A$ & $D-\mathrm{H} \cdots A$ \\
\hline $\mathrm{C} 6-\mathrm{H} 6 A \cdots \mathrm{O} 1^{\mathrm{ii}}$ & 0.97 & 2.46 & $3.334(2)$ & 150 \\
$\mathrm{C} 6-\mathrm{H} 6 B \cdots \mathrm{O} 1^{\mathrm{iii}}$ & 0.97 & 2.57 & $3.313(2)$ & 134 \\
$\mathrm{C} 8-\mathrm{H} 8 \cdots \mathrm{O} 3^{\mathrm{iii}}$ & 0.93 & 2.50 & $3.251(2)$ & 138
\end{tabular}

Symmetry codes: (ii) $-x+2,-y+1,-z$; (iii) $-x+5 / 2, y, z-1 / 2$. 